(c) Elsevier/INRA

Article original

\title{
Paramètres du croisement entre 3 souches de lapin et analyse de la réponse à une sélection sur la taille de portée : caractères des portées à la naissance et au sevrage
}

\author{
JM Brun \\ INRA, station d'amélioration génétique des animaux, \\ BP 27, 31326 Castanet-Tolosan, France \\ (Reçu le 30 décembre 1991; accepté le 30 juin 1993)
}

\begin{abstract}
Résumé - Une expérience de croisement à double étage a été réalisée entre trois souches de lapins de l'INRA, une souche d'origine raciale néo-zélandaise blanche maintenue sans sélection (A), et deux souches sélectionnées depuis 13 générations sur la taille de portée, l'une $\left(\mathrm{A}^{\prime}\right)$ dérivée de $\mathrm{A}$ et l'autre $(\mathrm{B})$ d'origine raciale californienne. Les caractères de 1853 portées de rang 1 à 3 et issues de 9 types génétiques de lapines sont analysés : nombre de lapereaux nés totaux (NT), nés vivants (NV), sevrés (NS), poids de la portée (PPS) et poids moyen du lapereau au sevrage (PIS). Les effets génétiques additifs directs, maternels, grand-maternels, ainsi que les effets d'hétérosis directs et maternels sur ces caractères, considérés comme des attributs de la portée, sont estimés. On met en évidence des effets additifs directs significatifs sur NT : les gènes B diminuent la viabilité avant la naissance des lapereaux qui les portent. La souche A exerce un effet maternel défavorable sur N'T, NV et NS mais non sur PPS, du fait d'un effet favorable sur PIS. Les effets d'hétérosis directs sont en général non significatifs sauf sur NS et PPS dans le croisement $A \times B(6 \%)$. En revanche, les effets d'hétérosis maternels sur les tailles et poids de portée sont importants, de l'ordre de $15 \%$ quand les deux souches en présence n'ont pas la même origine raciale et de $7 \%$ entre les souches $\mathrm{A}$ et $\mathrm{A}^{\prime}$. La sélection en souche fermée $\mathrm{A}^{\prime}$ s'est traduite par un progrès génétique qui, mesuré en souche pure, est relativement faible $(+0,6 \mathrm{NT},+0,4 \mathrm{NS})$ mais qui s'amplifie en croisement avec la souche $\mathrm{B}:$ la différence entre la moyenne des métisses réciproques $A^{\prime} \times B$ et celle des métisses $A \times B$ est de $1,0 \mathrm{NT}$ et $0,8 \mathrm{NS}$. Cette différence d'expression du progrès génétique en pur et en croisement pourrait s'expliquer par un effet du contexte génétique (homozygote/hétérozygote) sur l'expression de la variabilité génétique. La sélection sur la taille de portée a augmenté la composante maternelle de la valeur de la souche $\mathrm{A}^{\prime}(+1,0 \mathrm{NT},+0,7 \mathrm{NS})$ avec une tendance à la diminution de la composante directe $(-0,4 \mathrm{NT},-0,3 \mathrm{NS})$ liée aux gènes de viabilité des lapereaux.
\end{abstract}

lapin / paramètre de croisement / réponse à la sélection / caractère de portée 
Summary - Crossbreeding parameters between 3 rabbit strains and analysis of the response to selection for litter size: litter traits at birth and weaning. A crossbreeding experiment involved 3 INRA rabbit strains, an unselected strain (A) descending from the New Zealand white breed and 2 selected strains, one $\left(A^{\prime}\right)$ deriving from $A$ and the other from $B$, the Californian breed, both selected for litter size during 13 generations. Data from 1853 litters representing 9 breed types of dams over the first 3 parities were analysed: litter size at birth (total born (NBT) and born alive (NBA)) and at weaning $(N W)$, litter weight $(L W W)$ and average rabbit weight $(I W W)$ at weaning. Direct, maternal and grandmaternal additive genetic effects together with direct and maternal heterosis effects for these traits, considered as litter traits, were estimated. Significant direct additive genetic effects on NBT were shown: genes from strain $B$ decreased prenatal viability of the rabbits bearing them. Strain A had unfavourable maternal effects on litter size but not on $L W W$, because of a positive effect on IWW. Direct heterosis effects were generally not significant except for $N W$ and $L W W$ in the $\mathrm{A} \times \mathrm{B}$ cross (6\%). Conversely, maternal heterosis effects on litter size and weight were important, averaging $15 \%$ between strains of different breeds and $7 \%$ between strains $A$ and $A^{\prime}$. Selection response in the closed $A^{\prime}$ strain was small when measured in purebreds $(+0.6 \mathrm{NBT},+0.4 \mathrm{NW})$ but was larger in crosses with strain $B$; the difference between the average of reciprocal crossbred dams $\mathrm{A}^{\prime} \times \mathrm{B}$ and $\mathrm{A} \times \mathrm{B}$ was $1.0 \mathrm{NBT}$ and $0.8 \mathrm{NW}$. Such a different expression of the genetic gain in purebred and crossbred dams might be due to an effect of the genetic background (homozygous vs heterozygous) on the expression of genetic variability. Selection on litter size increased the maternal component of the genetic value of strain $A^{\prime}(+1.0 \mathrm{NBT},+0.7 \mathrm{NW})$ but tended to decrease the individual component $(-0.4 \mathrm{NBT},-0.3 \mathrm{NW})$, linked to the viability genes of the kids.

rabbit / crossbreeding parameter / selection response / litter trait

\section{INTRODUCTION}

Le schéma national d'amélioration génétique du lapin mis en place par l'INRA dans les années 1970 repose sur l'utilisation d'un croisement à double étage pour produire les lapereaux à viande. Ceux-ci résultent du croisement entre des mâles de souches à aptitudes bouchères avec des femelles métisses issues du croisement entre souches de l'INRA à bonnes aptitudes reproductives, les souches A1066 d'origine californienne et A1077 d'origine néo-zélandaise blanche. Depuis 1976, ces dernières sont sélectionnées sur la taille de portée par sélection intra-population (Matheron et Rouvier, 1977). La souche A1077 est sélectionnée en présence d'une souche témoin, la souche A9077, issue du même pool génétique (Matheron et Chevalet, 1977). La réponse à la sélection a été mesurée en souche pure A1077 après 9 générations (Matheron et Poujardieu, 1984).

Qu'en est-il de la réponse corrélative au niveau des femelles croisées? Dans le but de l'évaluer, une expérience de comparaison entre des femelles métisses issues de parents sélectionnés ou non sélectionnés a été réalisée. Cette expérience de croisement, où les souches A1066, A1077 et A9077 jouent en fait des rôles symétriques, permet aussi d'estimer leurs paramètres génétiques en croisement et d'analyser l'effet de la sélection en termes de réponse sur les effets génétiques directs et indirects. Dans la suite du texte, les souches A9077, A1077 et A1066 seront 
respectivement appelées $\mathrm{A}, \mathrm{A}^{\prime}$ et $\mathrm{B}$, ce qui permet de distinguer à la fois leur origine raciale $\left(A\right.$ et $A^{\prime}$ contre $B$ ) et leur histoire selective ( $A^{\prime}$ dérive de $A$ par sélection).

\section{MATÉRIEL ET MÉTHODES}

\section{Animaux et protocole expérimental}

L'expérience a eu lieu entre les mois de mai 1987 et juin 1989 à l'élevage expérimental de l'INRA de Toulouse. Elle s'est déroulée en 3 étapes (tableau I). Lors de la première étape est réalisé un plan de croisement factoriel $3 \times 3$ entre les trois souches. Chaque souche est représentée par 70 femelles et 10 mâles, issus de la $13^{\mathrm{e}}$ génération pour les souches sélectionnées et de la $8^{\mathrm{e}}$ pour la souche témoin. Lors de la $2^{\mathrm{e}}$ étape, les 9 types génétiques de lapines issues de l'étape 1, 3 purs et 6 métisses, sont croisés avec des mâles des trois souches étudiées selon un plan factoriel incomplet. Une partie de cette étape constitue une répétition de l'étape 1. Enfin, les 9 types génétiques de lapines utilisés à l'étape 2 sont de nouveau étudiés lors d'une $3^{e}$ étape, en croisement avec 2 souches de mâles de croisement terminal appelées $\mathrm{C}_{1}$ et $\mathrm{C}_{2}$. À chaque étape, le plan d'accouplement consiste en groupes de reproduction qui restent stables tout au long de l'étape : ces groupes de reproduction comportent 1 mâle et, selon l'étape, entre 5 et 9 femelles, avec le souci d'équilibre entre les types génétiques de femelles affectés à un mâle donné. Un stock de mâles de remplacement permet de remédier à la mort ou à l'élimination des mâles titulaires. À chaque étape, les lapines sont contrôlées sur leurs 3 premières portées. Elles seront ensuite autopsiées au $15^{\mathrm{e}} \mathrm{j}$ de gestation de leur $4^{\mathrm{e}}$ portée, pour le contrôle du taux d'ovulation et de la viabilité embryonnaire. Les conditions de conduite de l'élevage sont les suivantes : la mise à la reproduction des lapines a lieu à l'âge de $120 \mathrm{j}$. Ensuite, les lapines sont représentées au mâle $10 \mathrm{j}$ après la mise bas. Un diagnostic de gestation est réalisé par palpation $14 \mathrm{j}$ après la saillie et les lapines sont présentées au mâle le lendemain en cas de saillie négative. Le sevrage des lapereaux se fait à $30 \mathrm{j}$ d'âge.

\section{Caractères analysés}

Nous avons analysé, sur la population des portées sevrées, les nombres de lapereaux nés totaux, nés vivants, sevrés, le poids total de la portée au sevrage et le poids individuel moyen du lapereau sevré. Ces caractères sont considérés comme des attributs de la portée et non de la mère.

\section{Analyses statistiques}

\section{Estimation des valeurs des types génétiques de portée}

Le dispositif expérimental présente une disconnexion entre les étapes 1 et 2 d'une part et l'étape 3 d'autre part. Nous avons analysé ces deux ensembles séparément selon les deux modèles suivants d'analyse de variance à effets fixés : 
Tableau I. Nombre de portées contrôlées par type génétique à chaque étape du plan de croisement.

\begin{tabular}{|c|c|c|c|c|c|c|c|c|c|c|c|c|}
\hline & \multicolumn{2}{|c|}{ Mâles ${ }^{1}$} & \multicolumn{10}{|c|}{ Femelles ${ }^{1}$} \\
\hline & Type & $N_{m}^{2}$ & & $A$ & $B$ & $A^{\prime}$ & $B A^{\prime}$ & $A^{\prime} B$ & $A^{\prime} A$ & $A A^{\prime}$ & $A B$ & $B A$ \\
\hline & & & $\mathrm{N}_{f}^{2}:$ & 70 & 70 & 70 & & & & & & \\
\hline Étape 1 & $\begin{array}{l}\mathrm{A} \\
\mathrm{B} \\
\mathrm{A}^{\prime}\end{array}$ & $\begin{array}{l}10 \\
10 \\
10\end{array}$ & & $\begin{array}{l}61 \\
51 \\
52\end{array}$ & $\begin{array}{l}45 \\
53 \\
44\end{array}$ & $\begin{array}{l}43 \\
45 \\
53\end{array}$ & & & & & & \\
\hline Étape 2 & $\begin{array}{l}\mathrm{A} \\
\mathrm{B} \\
\mathrm{A}^{\prime}\end{array}$ & $\begin{array}{l}26 \\
26 \\
26\end{array}$ & $\mathrm{~N}_{f}^{2}:$ & $\begin{array}{l}78 \\
55 \\
62 \\
66\end{array}$ & $\begin{array}{l}78 \\
63 \\
60 \\
48\end{array}$ & $\begin{array}{l}78 \\
56 \\
59 \\
66\end{array}$ & $\begin{array}{c}26 \\
58 \\
- \\
-\end{array}$ & $\begin{array}{c}26 \\
55 \\
- \\
-\end{array}$ & $\begin{array}{c}26 \\
- \\
70 \\
-\end{array}$ & $\begin{array}{c}26 \\
- \\
70 \\
-\end{array}$ & $\begin{array}{c}26 \\
- \\
- \\
64\end{array}$ & $\begin{array}{c}26 \\
- \\
- \\
69\end{array}$ \\
\hline Étape 3 & $\begin{array}{l}\mathrm{C}_{1} \\
\mathrm{C}_{2}\end{array}$ & $\begin{array}{l}12 \\
12\end{array}$ & $\mathrm{~N}_{f}^{2}:$ & $\begin{array}{l}24 \\
27 \\
38\end{array}$ & $\begin{array}{l}24 \\
24 \\
20\end{array}$ & $\begin{array}{l}24 \\
31 \\
31\end{array}$ & $\begin{array}{l}24 \\
27 \\
28\end{array}$ & $\begin{array}{l}24 \\
25 \\
35\end{array}$ & $\begin{array}{l}24 \\
21 \\
27\end{array}$ & $\begin{array}{l}24 \\
29 \\
25\end{array}$ & $\begin{array}{l}24 \\
29 \\
26\end{array}$ & $\begin{array}{l}24 \\
28 \\
24\end{array}$ \\
\hline
\end{tabular}

${ }^{1}$ Pour les types métisses, le type génétique du père est donné en premier; ${ }^{2} \mathrm{~N}_{m}$ et $\mathrm{N}_{f}$ désignent le nombre de mâles et de femelles respectivement.

- Modèle 1 : sur les étapes 1 et 2 , avec les effets fixés «génotype de la portée» (15 niveaux), «étape» ( 2 niveaux), «numéro de portée» ( 3 niveaux) et les interactions entre ces effets 2 à 2 .

- Modèle 2 : sur l'étape 3, avec les effets fixés «génotype de la mère» (9 niveaux), «génotype du père» (2 niveaux), «numéro de portée» (3 niveaux) et les interactions entre ces effets.

Les variables «poids de portée au sevrage» et «poids individuel moyen du lapereau sevré» ont également été analysées en introduisant la taille de portée au sevrage comme covariable selon un modèle de régression quadratique avec des coefficients propres à chaque génotype.

\section{Estimation des paramètres du croisement}

Les paramètres du croisement qui sont estimés sont ceux du modèle de Dickerson (1969) : les effets génétiques additifs direct, maternel et grand-maternel $\left(g^{\mathrm{I}}, g^{\mathrm{M}}\right.$ et $g^{\mathrm{N}}$ respectivement) ainsi que les effets d'hétérosis direct et maternel ( $h^{\mathrm{I}}$ et $h^{\mathrm{M}}$ respectivement). Ils sont estimés à partir des estimées des performances moyennes des types génétiques de portées selon la méthode des moindres carrés généralisés exposée par Fimland (1985) et Eisen (1989). Cette estimation globale des paramètres génétiques n'est pas affectée par la disconnexion entre étapes sous l'hypothèse d'absence d'interaction entre les effets génétiques et l'étape de l'expérience. Le modèle d'estimation des paramètres s'écrit :

$$
\mathbf{y}=\mathbf{K} \mathbf{p}+\mathbf{e}
$$

où $\mathbf{y}$ désigne le vecteur des estimées des performances moyennes des types génétiques, $\mathbf{p}$, le vecteur des paramètres génétiques et $\mathbf{K}$, la matrice de décomposition 
des valeurs moyennes des types génétiques en fonction de $\mathbf{p}$ (tableau II) avec $\operatorname{var}(\mathbf{e})=\mathbf{V}$, où $\mathbf{V}$ désigne la matrice des variances et covariances des estimées des types génétiques. Les solutions du système [1] sont :

$$
\widehat{\mathbf{p}}=\left(\mathbf{K}^{\prime} \mathbf{V}^{-1} \mathbf{K}\right)^{-1} \mathbf{K}^{\prime} \mathbf{V}^{-1} \mathbf{y} \text { avec } \operatorname{var}(\widehat{\mathbf{p}})=\left(\mathbf{K}^{\prime} \mathbf{V}^{-1} \mathbf{K}\right)^{-1}
$$

Les 18 types génétiques de la bande 3 ont été ramenés à 9 en considérant comme une souche unique (appelée « $\mathrm{C}$ ) les deux souches de mâles de croisement terminal. Cette simplification est autorisée par l'absence d'interaction entre le génotype de la mère et le génotype du père dans le modèle 2 et la distribution identique des génotypes de pères dans les différents génotypes de mère. Les valeurs moyennes de ces 9 types génétiques fictifs sont estimées par l'effet du type génétique de la mère du modèle 2. L'effet $g_{c}^{I}$ n'a donc pas de signification génétique et d'autant moins qu'il est confondu avec les effets de milieu propres à l'étape 3.

Le modèle [1] est reparamétré pour estimer deux contrastes $\left(g_{\mathrm{B}}-g_{\mathrm{A}}\right)$ et $\left(g_{\mathrm{A}^{\prime}}-g_{\mathrm{A}}\right)$ pour chacun des trois types d'effets additifs ainsi que les contrastes $\left(h_{\mathrm{CB}}^{\mathrm{I}}-h_{\mathrm{CA}}^{\mathrm{l}}\right)$ et $\left(h_{\mathrm{CA}^{\prime}}^{\mathrm{I}}-h_{\mathrm{CA}}^{\mathrm{I}}\right)$. Ces derniers sont des combinaisons d'effets d'hétérosis direct obtenus avec les 2 souches de mâles de croisement terminal (les souches $C_{1}$ et $C_{2}$ ) et n'ont donc pas de réelle signification génétique.

\section{RÉSULTATS}

\section{Souches pures, croisements simples et croisements à double étage}

Les résultats zootechniques de l'analyse de cette expérience de croisement ont été publiés par ailleurs (Brun, 1990). Nous nous limiterons ici à donner les estimées des moyennes des types génétiques des portées (tableaux III et IV) et à souligner les points essentiels concernant les effets du croisement et de la sélection.

Le croisement simple n'augmente les tailles de portées que pour les mères de type $\mathrm{B}$ : accouplées avec des mâles $\mathrm{A}$ ou $\mathrm{A}^{\prime}$, elles donnent plus de lapereaux nés totaux, et avec des mâles $A$ plus de nés vivants et de sevrés également. Les lapines de souches $A$ et $A^{\prime}$, en revanche, ont la même prolificité en pur et en croisement simple, même avec des mâles $\mathrm{B}$, d'origine raciale différente. Contrairement au croisement simple, le croisement à double étage utilisant une lapine croisée modifie sensiblement les caractères des portées. Cela laisse prévoir, d'une part, l'importance de la composante d'effet maternel dans la détermination de la taille de portée et, d'autre part, un déterminisme non additif de celle-ci.

L'effet de la sélection pratiquée dans la souche $\mathrm{A}^{\prime}$ peut s'évaluer, en écart à la souche témoin A, pour deux modes d'utilisation de la souche (tableau V) : comme femelles pures utilisées en pur ou comme composante d'une femelle métisse obtenue en croisement avec la souche B et utilisée en croisement terminal. Les femelles pures de la souche sélectionnée font naître et sèvrent plus de lapereaux que celles de la souche témoin $(0,6$ à la naissance et 0,4 au sevrage $)$ mais cette supériorité n'est pas significative. Ces lapereaux sont plus légers au sevrage $(-40 \mathrm{~g})$.

L'écart observé entre les femelles métisses demi-sang $B$ issues de la souche sélectionné $\left(\mathrm{BA}^{\prime}\right.$ et $\mathrm{A}^{\prime} \mathrm{B}$ ) et celles issues de la souche témoin ( $\mathrm{BA}$ et $\mathrm{AB}$ ) est 


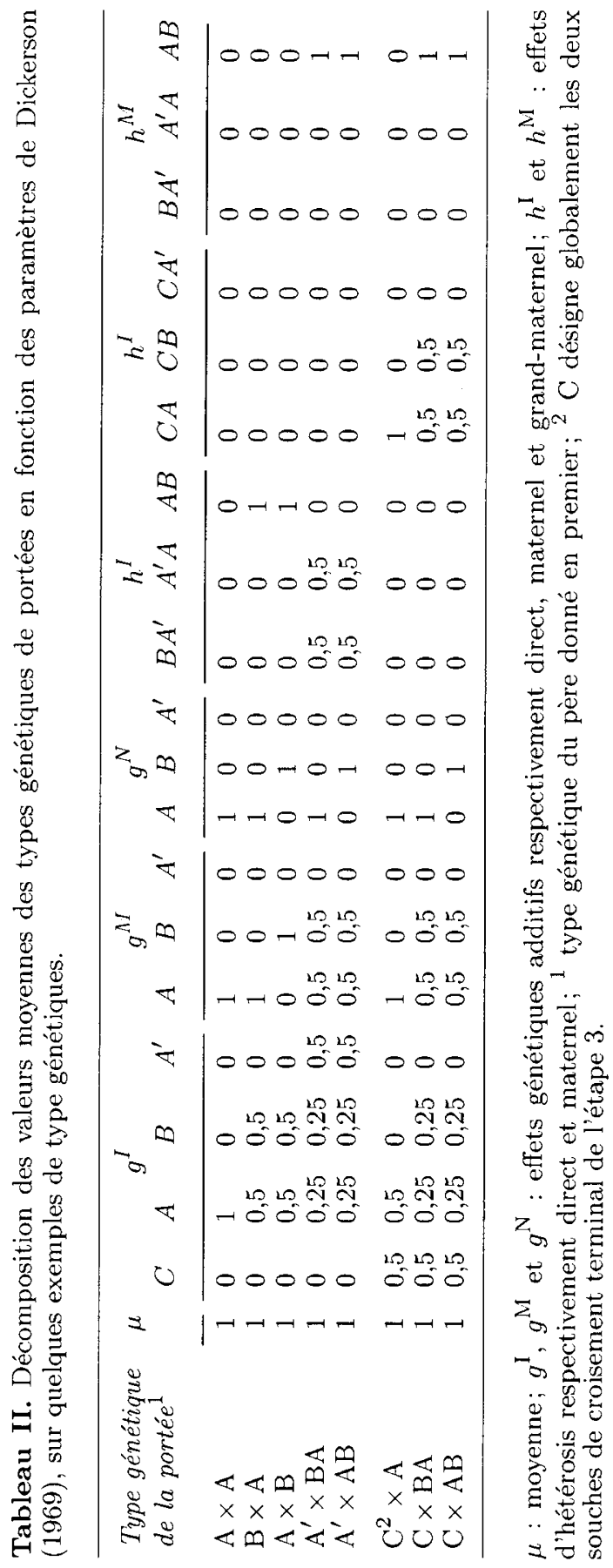


Paramètres du croisement chez le lapin

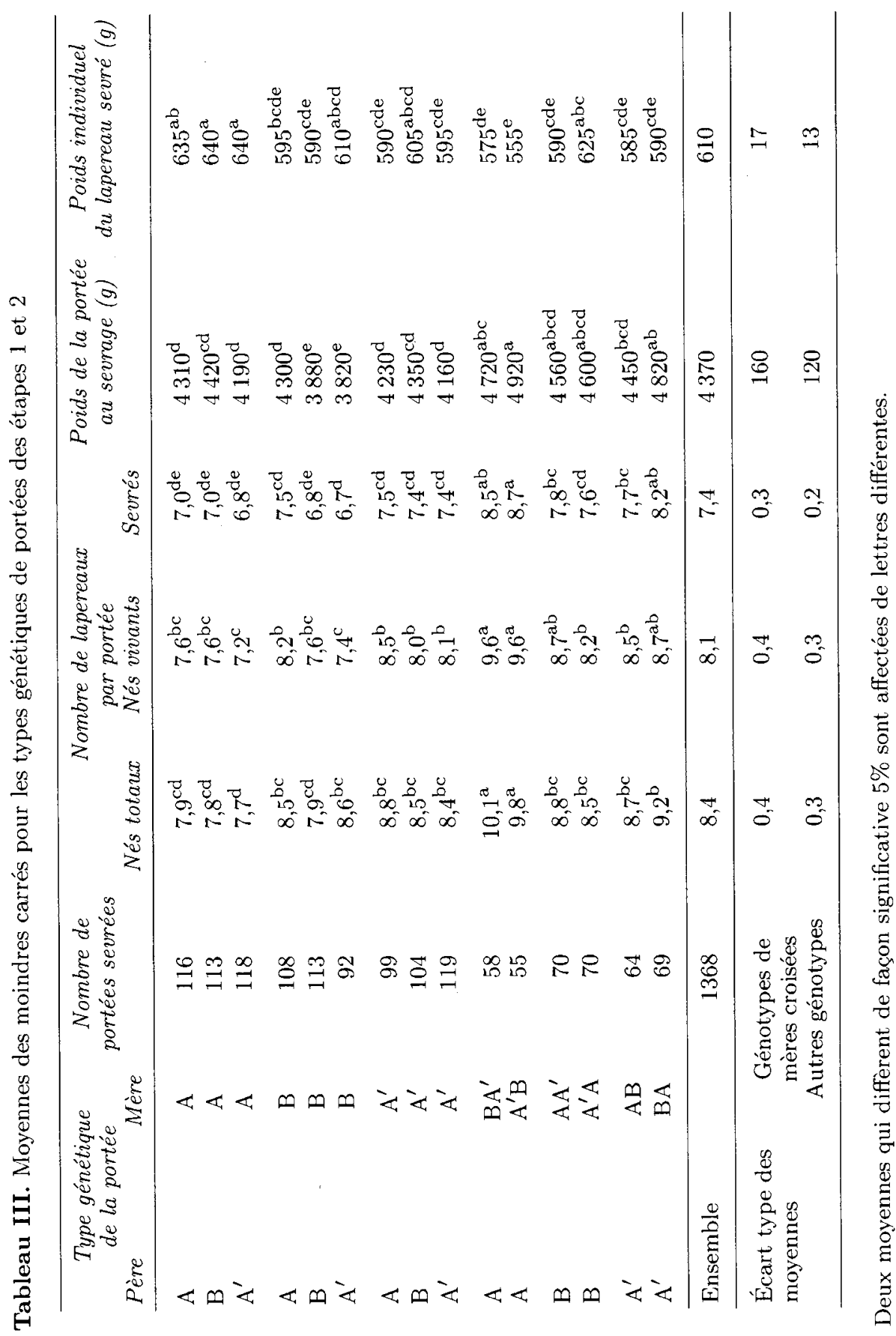




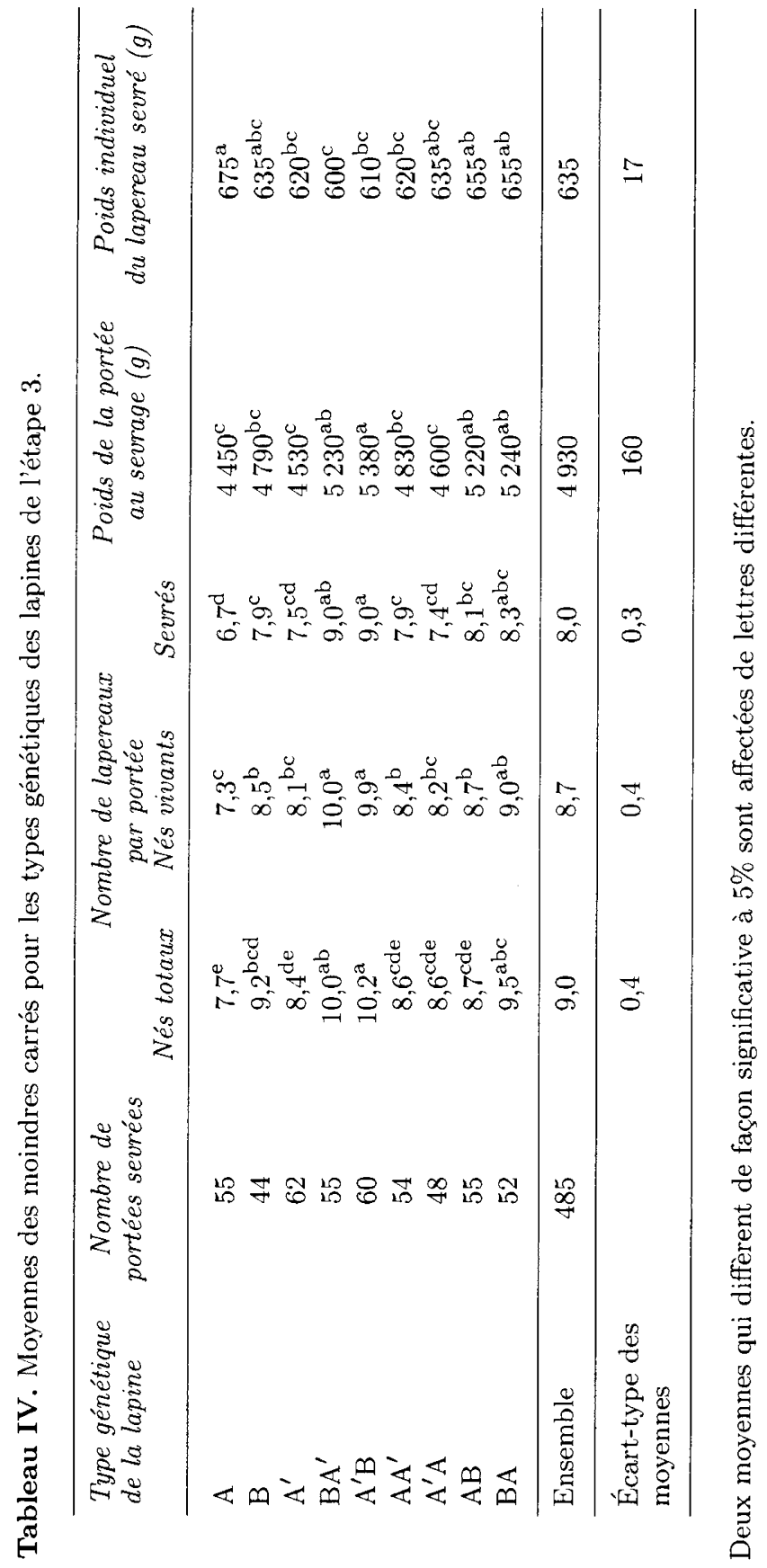


Tableau V. Effets de la sélection dans la souche $\mathrm{A}^{\prime}$ en fonction de son mode d'utilisation, en écart à la souche témoin $\mathrm{A}$.

\begin{tabular}{|c|c|c|c|c|c|}
\hline \multirow[t]{2}{*}{$\begin{array}{l}\text { Mode d'utilisation } \\
\text { de la souche } A^{\prime}\end{array}$} & \multicolumn{3}{|c|}{$\begin{array}{c}\text { Nombre de } \\
\text { lapereaux } \\
\text { par portée }\end{array}$} & \multirow[t]{2}{*}{$\begin{array}{c}\text { Poids } \\
\text { de la portée } \\
\text { au sevrage (g) }\end{array}$} & \multirow[t]{2}{*}{$\begin{array}{c}\text { Poids moyen } \\
\text { du lapereau } \\
\text { sevré }(g)\end{array}$} \\
\hline & Nés totaux & Nés vivants & Sevrés & & \\
\hline En pur ${ }^{1}$ & $0,6 \pm 0.4$ & $0,5 \pm 0,4$ & $0,4 \pm 0,3$ & $-150 \pm 170$ & $-40 \pm 18^{*}$ \\
\hline $\begin{array}{l}\text { Comme compo- } \\
\text { sante d'une } \\
\text { lapine métisse }\end{array}$ & $1,0 \pm 0,4^{* *}$ & $1,1 \pm 0,4^{* *}$ & $0,8 \pm 0,3^{* *}$ & $75 \pm 160$ & $-48 \pm 17^{* *}$ \\
\hline
\end{tabular}

${ }^{1}$ Cette ligne correspond au contraste $\mathrm{A}^{\prime} \times \mathrm{A}^{\prime}-\mathrm{A} \times \mathrm{A}$ (tableau III); ${ }^{2}$ Cette ligne correspond au contraste $0,5\left(\mathrm{C} \times \mathrm{A}^{\prime} \mathrm{B}+\mathrm{C} \times \mathrm{BA}^{\prime}-\mathrm{C} \times \mathrm{AB}-\mathrm{C} \times \mathrm{BA}\right)($ tableau IV).

nettement supérieur à l'écart attendu sous l'hypothèse où le progrès génétique réalisé dans la souche $\mathrm{A}^{\prime}$ se transmet de façon additive aux filles croisées, auquel cas l'écart observé serait de l'ordre de 0,2 à 0,3 lapereau par portée.

\section{Paramètres génétiques du croisement entre les trois souches (tableaux VI et VII)}

\section{Effets liés aux gènes des lapereaux}

Il apparaît un effet direct significatif $(P<0,10)$ sur le nombre de lapereaux nés totaux. Il témoigne d'un effet additif des gènes des embryons sur leur viabilité. Les gènes $B$ ont ainsi un effet défavorable, par rapport aux gènes $A$, sur la viabilité embryonnaire. Cela se traduit par une diminution significative de la taille de portée à la naissance, de l'ordre de 0,8 laperau né total. Cet effet défavorable se prolonge jusqu'au sevrage où il n'est toutefois pas significatif. Les gènes $A^{\prime}$ ont également tendance à diminuer, relativement aux gènes $\mathrm{A}$, la viabilité des embryons qui les portent.

Comme les effets additifs directs, les effets d'hétérosis directs sur les tailles et poids des portées apparaissent parfois significatifs : de 5 à $6 \%$ sur la taille et le poids de portée au sevrage dans le croisement $\mathrm{A} \times \mathrm{B}$. Dans les croisements $\mathrm{A}^{\prime} \times \mathrm{B}$ et $\mathrm{A} \times \mathrm{B}$ on note également un hétérosis significatif sur le poids de portée ajusté pour la taille, traduisant un effet d'hétérosis sur la vitesse de croissance des lapereaux (de l'ordre de $3 \%$ ).

\section{Effets liés aux gènes des mères}

Les effets additifs maternels sur les tailles de portées présentent des différences entre souches qui sont fréquemment significatives. La souche $\mathrm{A}^{\prime}$ montre une supériorité d'effet maternel sur la souche $\mathrm{A}$ à tous les stades de la portée. La souche B présente une supériorité du même ordre sur le nombre de nés totaux mais la perd ensuite à cause d'un effet maternel défavorable sur la viabilité des lapereaux à la naissance. Le classement des souches pour leur effet maternel est différent pour la taille et pour 


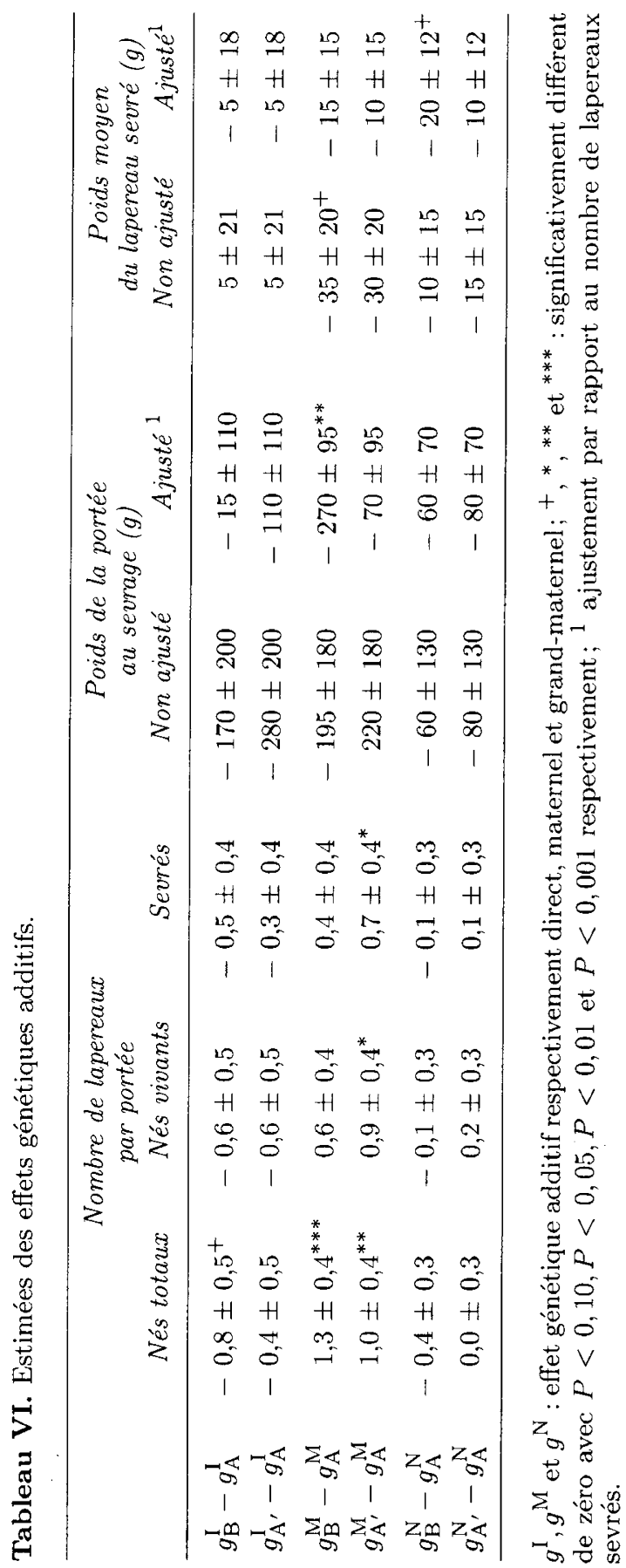




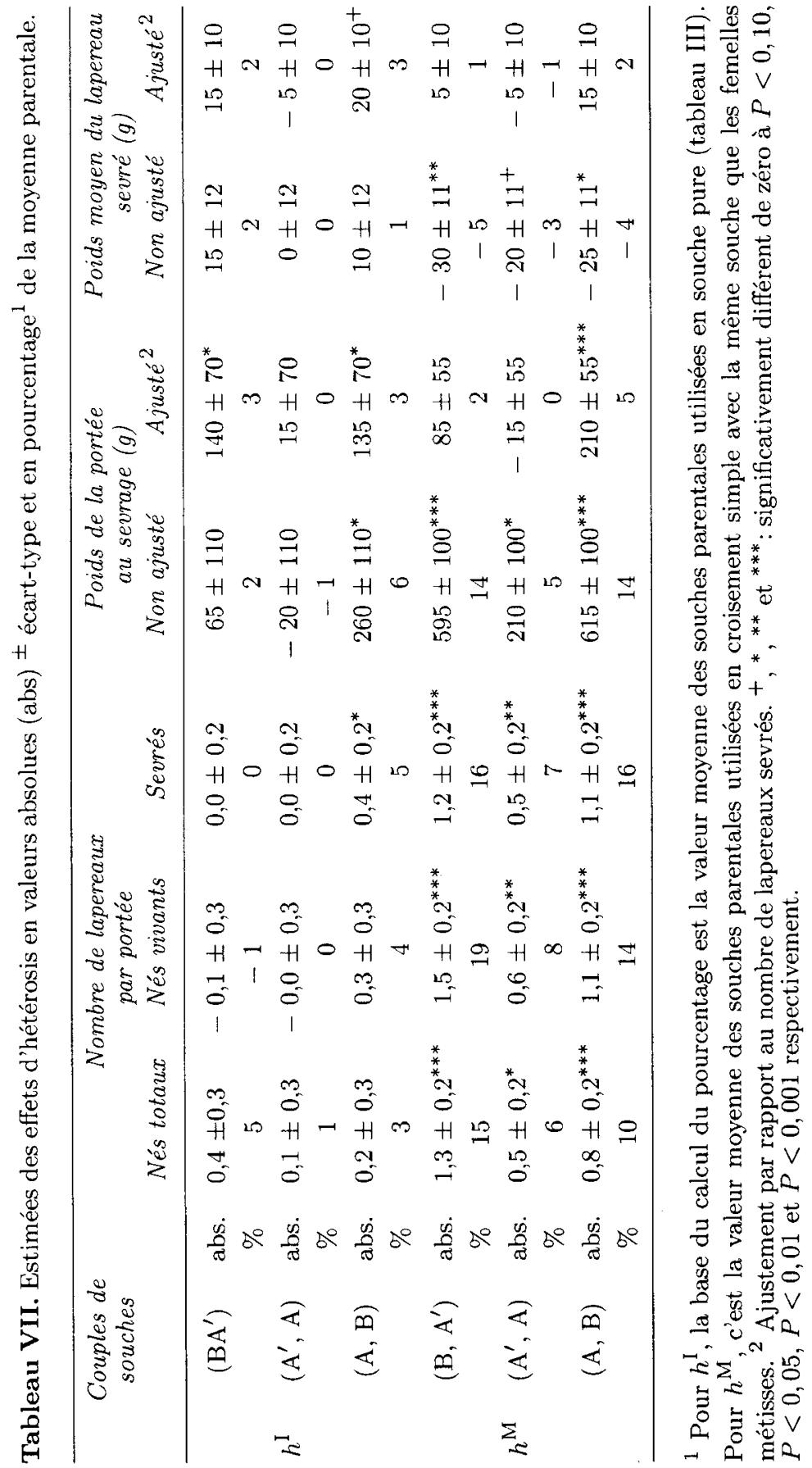


le poids de portée au sevrage en raison d'effets maternels spécifiques sur le poids du lapereau au sevrage. La souche B exerce un effet maternel défavorable sur ce caractère. Le poids de portée au sevrage ajusté montre une infériorité maternelle très significative de la souche $\mathrm{B}$, traduisant vraisemblablement une moins bonne aptitude laitière.

Les effets d'hétérosis maternels sur les tailles de portées et sur le poids de portée au sevrage non ajusté sont tous significatifs et varient, selon le couple de souches considéré et le caractère, entre 5 et 19\%. L'hétérosis maximal s'obtient lorsque les 2 souches en présence ont des origines raciales différentes. Sur les tailles de portées à la naissance, le couple $\left(\mathrm{A}^{\prime}, \mathrm{B}\right)$ donne cependant plus d'hétérosis $(P<0,10)$ que le couple $(A, B)$. On note un effet d'hétérosis maternel significatif sur le poids de portée ajusté dans le croisement $\mathrm{A} \times \mathrm{B}$.

On ne relève aucun effet grand-maternel significatif avec cependant une tendance négative pour celui de la souche $B$; cet effet apparaît plus nettement dans la différence entre les femelles métisses $\mathrm{BA}$ et $\mathrm{AB}$ qu'entre les métisses $\mathrm{BA}^{\prime}$ et $\mathrm{A}^{\prime} \mathrm{B}$. Cela semble indiquer que ces effets sont liés aux différences de taille de portée d'origine des lapines en fonction de la souche de leur mère : l'écart moyen de taille de portée d'origine entre lapines $\mathrm{BA}$ et $\mathrm{AB}$ est de l'ordre de 0,7 lapereau; celui qui existe entre lapines $\mathrm{BA}^{\prime}$ et $\mathrm{A}^{\prime} \mathrm{B}$ est de l'ordre de 0,1 lapereau.

\section{DISCUSSION}

Le modèle statistique à effets fixés qui est utilisé pour estimer les effets des types génétiques n'est pas optimal. On considère en effet que les observations sont indépendantes alors qu'il existe entre elles des corrélations liées à l'apparentement des animaux et à la répétition des performances d'une même lapine. La non-prise en compte des apparentements fait que les estimées des performances moyennes des types génétiques ne sont plus les meilleures estimations linéaires sans biais de ces effets (Komender et Hoeschele, 1989). La prise en compte des parentés, mais aussi des performances répétées, se heurte aux différences de structure de la variabilité génétique entre types génétiques et notamment entre types génétiques purs et croisés $F_{1}$ issus de deux populations (Brun et Rouvier, 1981) qui rendent difficile à admettre l'hypothèse d'une variance génétique additive unique.

Le progrès génétique observé en souche pure $\mathrm{A}^{\prime}$ est faible par rapport au progrès espéré au départ de l'expérience de sélection et même par rapport au progrès réalisé à la $9^{\text {e }}$ génération de sélection (Matheron et Poujardieu, 1984). Le progrès escompté sur la base d'une extrapolation linéaire du progrès réalisé à G9 est de 1,9 lapereau né total et 1,0 sevré au lieu des valeurs de 0,6 et 0,4 qui sont observées ici.

Le modèle génétique utilisé (Dickerson, 1969) nous permet d'analyser l'effet de la sélection en termes de réponse sur les effets génétiques additifs directs et indirects. Selon ce modèle, la valeur globale d'une souche, $g^{T}$, est la somme de trois composantes : les valeurs additives directe, maternelle et grand-maternelle : $g^{\mathrm{T}}=g^{\mathrm{I}}+g^{\mathrm{M}}+g^{\mathrm{N}}$. L'écart $\Delta g^{\mathrm{T}}=g_{\mathrm{A}^{\prime}}^{\mathrm{T}}-g_{\mathrm{A}}^{\mathrm{T}}$ est la somme des écarts $\Delta g^{\mathrm{I}}, \Delta g^{\mathrm{M}}$ et $\Delta g^{\mathrm{N}}$ donnés au tableau VI. En ce qui concerne la valeur additive directe, l'écart de la souche sélectionnée par rapport au témoin est de $-0,4$ lapereau né total, $-0,6$ né vivant, $-0,3$ sevré, $-280 \mathrm{~g}$ de poids de portée sevrée et $-110 \mathrm{~g}$ de poids de portée ajusté pour le nombre de lapereaux sevrés. Ces écarts, en général non 
significatifs, indiquent une tendance à la diminution de la valeur additive directe de la souche sélectionnée pour la viabilité des embryons et des lapereaux et en particulier pour la viabilité périnatale. Par contre, la sélection a significativement augmenté la composante maternelle de la valeur de la souche pour le nombre de lapereaux, de l'ordre de 1 lapereau à la naissance et 0,7 au sevrage. On constate une tendance à une diminution de l'effet maternel sur le poids individuel moyen du lapereau sevré. L'absence de différence maternelle sur le poids total de portée à nombre constant de lapereaux semble indiquer une constance de la production laitière sous l'effet de la sélection pour la prolificité.

Outre ses effets sur les valeurs additives, la sélection de la souche A' a également modifié ses effets génétiques non additifs en croisement avec la souche B. L'hétérosis direct de l'ordre de 3 à $5 \%$ observé dans le croisement $\mathrm{A} \times \mathrm{B}$ sur les tailles de portée à la naissance et au sevrage n'existe, dans le croisement $\mathrm{A}^{\prime} \times \mathrm{B}$, que sur le nombre de lapereaux nés totaux. Cette différence est cohérente avec la disparition de l'hétérosis direct sur les nombres de lapereaux nés vivants et sevrés dans le croisement $\mathrm{A}^{\prime} \times \mathrm{B}$ depuis les expériences de croisement précédentes (Brun et Rouvier, 1984, 1988). Comme l'hétérosis direct, l'hétérosis maternel a également varié sous l'effet de la sélection, comme en témoigne, par exemple, sa supériorité sur la taille de portée à la naissance dans le croisement $\mathrm{A}^{\prime} \times \mathrm{B}$ par rapport au croisement $\mathrm{A} \times \mathrm{B}$. Cette différence pourrait être la contrepartie d'une plus forte dépression de consanguinité dans la souche $\mathrm{A}^{\prime}$ que dans la souche $\mathrm{A}$. Calculé en 1991, le coefficient moyen de consanguinité de la souche $\mathrm{A}^{\prime}$ est supérieur à celui de la souche $\mathrm{A}$, avec les valeurs respectives de 16 et $10 \%$ (B Poujardieu, communication personnelle). Cette différence résulte à la fois d'un renouvellement plus rapide de la souche $\mathrm{A}^{\prime}$ et du fait qu'elle est sélectionnée. Une sélection sur index combinant des performances d'apparentés serait particulièrement propice à une augmentation du coefficient de consanguinité (Wray et Thompson, 1990).

Depuis leur création en 1968, les souches A $^{\prime}$ et B ont fait l'objet de 3 expériences de croisement, échelonnées à une dizaine d'années d'intervalle entre 1970 et 1989. Lors de chaque expérience, ces souches sont étudiées en pur, en croisements simples mutuels ainsi qu'avec une troisième souche et en croisement à double étage. Du fait de l'absence de témoin, les valeurs des types génétiques ne sont pas comparables entre expériences mais on peut comparer les écarts entre types génétiques intraexpérience ou encore les paramètres du croisement. Sur l'exemple de la variable "nombre de lapereaux nés vivants», on peut observer (tableau VIII) que (i) l'écart entre les souches pures, initialement en faveur de la souche B, s'est inversé, (ii) le croisement simple sur les lapines $B$ augmente le nombre de nés vivants dans les trois expériences alors qu'il ne l'augmente que dans la première pour les lapines $\mathrm{A}^{\prime}$, et (iii) l'écart entre les femelles métisses et les femelles pures (hétérosis maternel) augmente constamment. Ces faits indiquent que la réponse à la sélection, mesurée en souche pure, est moins élevée dans la souche $B$ que dans la souche $A^{\prime}$. L'effectif plus faible de la souche $B$, entraînant une dépression de consanguinité plus forte, pourrait contribuer à expliquer ce résultat. L'augmentation de l'hétérosis maternel entre les 2 souches pourrait n'être que la contrepartie de la dépression de consanguinité affectant les souches pures. La disparition de l'effet du croisement simple dans la souche $\mathrm{A}^{\prime}$ semble indiquer que la viabilité des embryons ne serait plus dans cette souche un facteur limitant de la taille de portée. L'observation d'une liaison non 
linéaire entre le nombre d'embryons et le nombre d'ovules pondus (Bolet et al, 1988) semble indiquer que l'augmentation de la taille de portée se heurte dans la souche $\mathrm{A}^{\prime}$ à une limite biologique, comme par exemple la capacité utérine. Dans la souche $\mathrm{B}$, au contraire, la viabilité des embryons semble toujours constituer un facteur limitant de la taille de portée.

Tableau VIII. Moyennes du nombre de lapereaux nés vivants par portée (NV) lors de trois expériences de croisement entre les souches $A^{\prime}$ et $B$.

\begin{tabular}{|c|c|c|c|c|c|c|c|}
\hline \multirow{2}{*}{$\begin{array}{l}\text { Type génétique } \\
\text { de la lapine }\end{array}$} & \multirow{2}{*}{$\begin{array}{c}\text { Mode } \\
\text { d'accouplement }\end{array}$} & \multicolumn{2}{|c|}{$1970-72$} & \multicolumn{2}{|c|}{$1979-80$} & \multicolumn{2}{|c|}{$1987-89$} \\
\hline & & $n$ & $N V$ & $n$ & $N V$ & $n$ & $N V$ \\
\hline B & Souche pure & 107 & 7,3 & 50 & 7,8 & 113 & 7,5 \\
\hline B & Croisement & 144 & 7,8 & 150 & 8,6 & 244 & 8,0 \\
\hline $\mathrm{A}^{\prime}$ & Souche pure & 150 & 7,1 & 50 & 8,0 & 99 & 8,1 \\
\hline & Croisement & 213 & 7,9 & 150 & 8,0 & 243 & 8,2 \\
\hline $\mathrm{A}^{\prime} \mathrm{B}+\mathrm{BA}^{\prime}$ & Croisement & 118 & 7,9 & 200 & 8,9 & 228 & 9,7 \\
\hline
\end{tabular}

La différence d'expression du progrès génétique selon qu'il est mesuré en souche pure ou en croisement mérite une discussion particulière; l'écart entre la valeur des femelles de souches pures $\mathrm{A}$ et $\mathrm{A}^{\prime}$ est faible par rapport à l'écart observé entre leurs filles croisées demi-sang $B$. Remarquons d'abord que ce résultat est original par rapport à un ensemble d'expériences de sélection intra population revues par Brun (1985) où l'on observait dans tous les cas un parallélisme entre les réponses en pur et en croisement. Le modèle génétique de Dickerson permet certes de rendre compte des différences observées : l'écart à la relation $\Delta \mathrm{G}_{\mathrm{c}}=\Delta \mathrm{G}_{\mathrm{p}} / 2$ peut s'expliquer par l'écart $\mathrm{h}_{\mathrm{BA}^{\prime}}^{\mathrm{M}}-\mathrm{h}_{\mathrm{BA}}^{\mathrm{M}}$. En effet, le progrès mesuré au niveau des femelles croisées, supposées accouplées avec des mâles testeurs d'une souche $\mathrm{C}$,

$$
\Delta \mathrm{G}_{c}=0,5\left(\mathrm{C} \times \mathrm{BA}^{\prime}+\mathrm{C} \times \mathrm{A}^{\prime} \mathrm{B}-\mathrm{C} \times \mathrm{AB}-\mathrm{C} \times \mathrm{BA}\right)
$$

a pour espérance :

$$
0,25 \Delta g^{\mathrm{I}}+0,5 \Delta g^{\mathrm{M}}+0,5 \Delta g^{\mathrm{N}}+0,5\left(h_{\mathrm{CA}^{\prime}}^{\mathrm{I}}-h_{\mathrm{CA}}^{\mathrm{I}}\right)+h_{\mathrm{BA}^{\prime}}^{\mathrm{M}}-h_{\mathrm{BA}}^{\mathrm{M}} .
$$

Mesuré au niveau de femelles pures utilisées en croisement simple, ce progrès, $\Delta \mathrm{G}_{p}=\mathrm{C} \times \mathrm{A}^{\prime}-\mathrm{C} \times \mathrm{A}$, a pour espérance : $0,5 \Delta g^{\mathrm{I}}+\Delta g^{\mathrm{M}}+\Delta g^{\mathrm{N}}+\left(h_{\mathrm{CA}^{\prime}}^{\mathrm{I}}-h_{\mathrm{CA}}^{\mathrm{I}}\right)$.

On peut également donner une autre interprétation au phénomène observé d'une expression différente du progrès génétique en pur et en croisement. Cette interprétation fait appel à la notion d'interaction génotype $\times$ milieu génétique (Brun, 1982), selon laquelle une même structure génétique, les gènes des souches $\mathrm{A}$ ou $\mathrm{A}^{\prime}$, s'exprime différemment selon le contexte, reproduction en race pure ou en croisement. Utilisées en croisement avec la souche $B$, les souches $A$ et $A^{\prime}$ présentent une hétérozygotie plus grande de l'ensemble des locus, y compris de ceux qui ne sont pas directement impliqués dans la détermination du caractère quantitatif étudié mais qui conditionnent les caractères d'adaptation des individus 
(Orozco et Bell, 1974). Les métisses, mieux adaptées du fait d'une hétérozygotie supérieure, expriment mieux les différences génétiques existant entre les souches pures $A$ et $A^{\prime}$. Un tel effet du back-ground génétique (homozygote ou hétérozygote) sur l'expression de la variabilité génétique a été observé par Mukai et al (1966) -chez la drosophile et par Gallais (1984) chez la luzerne. Cette hypothèse aurait des implications sur la meilleure méthode de sélection des souches pures pour améliorer leur croisement. Le recours à des tests en croisement, comme c'est le cas dans la sélection recurrente et réciproque, serait alors nécessaire pour «libérer» la variabilité génétique que l'on veut exploiter.

\section{CONCLUSION}

Cette expérience de croisement entre trois souches a permis de mettre en évidence, en ce qui concerne les caractères des portées à la naissance et au sevrage, des différences génétiques significatives, d'origine additive et non-additive. Á l'inverse de la souche A, la souche B exerce des effets additifs directs défavorables mais des effets maternels favorables sur la taille de portée à la naissance. Si les effets d'hétérosis direct sont rarement significatifs, les effets d'hétérosis maternel sur les tailles et poids de portées dépassent fréquemment $10 \%$ : ces valeurs élevées s'expliquent autant par la distance génétique entre les souches parentales que par la dépression de consanguinité qui affecte ces souches d'effectif limité, fermées depuis une vingtaine de générations et éventuellement sélectionnées. La sélection dans la souche $\mathrm{A}^{\prime}$ s'est traduite par un progrès génétique qui, mesuré en souche pure, est relativement faible mais qui s'amplifie en croisement avec la souche B. Cette expression différente du progrès génétique en pur et en croisement peut s'interpréter, entre autres, par une expression différente de la variabilité génétique selon le degré d'hétérozygotie du back-ground génétique.

\section{REMERCIEMENTS}

L'auteur remercie le responsable et les techniciens de l'élevage expérimental de lapins de l'INRA de Toulouse, F Tudela, Y Andreuzza et J Falières pour la réalisation consciencieuse du protocole expérimental ainsi que les deux lecteurs anonymes de la revue pour leur lecture constructive qui a permis d'améliorer le manuscrit.

\section{RÉFÉRENCES}

Bolet G, Brun JM, Hulot F (1988) Relationships between ovulation rate and embryonic survival in various strains of rabbits. 4th World Rabbit Congress, Budapest, 10-14 October 1988, World Rabbit Science Association 2, 149-157 Brun JM (1982) Interactions géniteur $\times$ population des partenaires. I. Définition d'indicateurs. Ann Génét Sél Anim 14, 463-480

Brun JM (1985) Interactions géniteur $\times$ population des partenaires. III. Synthèse bibliographiques. Génét Sél Evol 17, 561-578

Brun JM (1990) Variabilité génétique et effet de la sélection dans le croisement de trois souches de lapin. 1. Caractères des portées à la naissance et au sevrage. 
$5^{\text {èmes }}$ Journées de la Recherche Cunicole en France, Paris, 12-13 décembre 1990, INRA-ITAVI, communication $\mathrm{n}^{\circ} 64$

Brun JM, Rouvier R (1981) Éléments de génétique quantitative d'une population $\mathrm{F}_{1}$ issue du croisement de deux populations. In : Variabilité génétique conjointe en pur et en croisement $F_{1}$ de deux populations. Application à la sélection animale. Toulouse 13-14 octobre 1981, INRA Publ, 1982, Les Colloques de l'INRA n ${ }^{\circ} 10$, $55-64$

Brun JM, Rouvier R (1984) Effets génétiques sur les caractères des portées de trois souches de lapins utilisées en croisement. Génét Sél Evol 16, 367-384

Brun JM, Rouvier R (1988) Paramètres génétiques des caractères de la portée et du poids de la mère dans le croisement de deux souches de lapins sélectionnées. Génét Sél Evol 20, 367-378

Dickerson GE (1969) Experimental approaches in utilizing breed resources. Anim Breed Abstr 37, 191-202

Eisen EJ (1989) Genetic models to predict crossbred performance : a review. Rev Brasil Genet 12, 13-26

Fimland E (1983) Methods of estimating the effects of heterosis. Z Tierz Zuchtungsbiol $100,3-8$

Gallais A (1984) An analysis of heterosis vs inbreeding effects with an antotetraploid cross-fertilized plant : médicago sativa L. Genetics 106, 123-137

Komender P, Hoeschele I (1989) Use of mixed-model methodology to improve estimation of crossbreeding parameters. Livest Prod Sci 21, 101-113

Matheron G, Chevalet C (1977) Conduite d'une population témoin de lapins. Évolution à court terme du coefficient de consanguinité selon le schéma d'accouplement. Ann Génét Sél Anim 9, 1-13

Matheron G, Rouvier R (1977) Optimisation du progrès génétique sur la prolificité chez la lapine. Ann Génét Sél Anim 9, 303-407

Matheron G, Poujardieu B (1984) Expérience de sélection de la taille de portée chez la lapine. $3^{\mathfrak{e}}$ Congrès mondial de Cuniculture. Rome. 4-8 avril 1984, World Rabbit Science Association, 1, 43-53

Mukai T, Yowhikawa I, Sano K (1966) The genetic structure of natural populations of Drosophila melanogaster. IV. Heterozygous effects of radiation induced mutations of viability in various genetic backgrounds. Genetics 53, 513-527

Orozco F, Bell AE (1974) Reciprocal recurrent selection compared to within-strain selection for increasing rate of egg lay of Tribolium under optimal and stress conditions. Genetics 77, 143-161

Wray NR, Thompson R (1990) Advances in selection theory. In : Proc $4^{\text {th }}$ World Cong on Genet Appl to Livest prod, Edinburgh, July 1990 